\title{
Autoimmune comorbidities in Hashimoto's thyroiditis: different patterns of association in adulthood and childhood/adolescence
}

\author{
R M Ruggeri', F Trimarchi², G Giuffrida', R Certo', E Cama ${ }^{1,3}$, A Campennì ${ }^{4}$, \\ A Alibrandi ${ }^{5}$, F De Luca $^{3}$ and M Wasniewska ${ }^{3}$ \\ ${ }^{1}$ Division of Endocrinology, Department of Clinical and Experimental Medicine, ${ }^{2}$ Accademia Peloritana dei \\ Pericolanti, ${ }^{3}$ Department of Adult and Development Age Human Pathology 'Gaetano Barresi', ${ }^{4}$ Departments of \\ Biomedical Sciences and Morpho-Functional Imaging, and ${ }^{5}$ Economics, University of Messina, Messina, Italy
}

\author{
Correspondence \\ should be addressed \\ to R M Ruggeri \\ Email \\ rmruggeri@unime.it
}

\begin{abstract}
Objective: Hashimoto's thyroiditis (HT), the most common autoimmune thyroid disease at any age, is often associated with other autoimmune diseases. The present study was aimed to describe the type and frequency of non-thyroidal autoimmune diseases (NTADs) in HT patients and to delineate the clinical pattern of diseases clustering in pediatric/adolescent and adult age.

Design: Cross-sectional study.

Methods: 1053 newly diagnosed HT patients (500 adults (467 F, mean age $40.2 \pm 13.7$ years) and 553 children/ adolescents (449 F, mean age $11.1 \pm 3.0$ years)) were evaluated for common NTADs by means of careful recording of medical history, physical examination and assessment of selected autoantibody profiles.

Results: The prevalence of associated NTADs was significantly higher in adults than that in pediatric/adolescent HT patients $(P<.0001)$. In addition, the number of adult patients suffering from two or more associated NTADs was significantly higher than that of children/adolescent $(P<0.0001)$. A female prevalence was evident in both cohorts, but was significant in the adults $(P<0.0001)$. The epidemiological distribution of NTADs was strongly different in the two cohorts, the most frequent associated diseases being arthropathies and connective tissue diseases in adults and type 1 diabetes and coeliac disease in children/adolescents. Skin diseases were represented with similar prevalence in both cohorts, vitiligo being the most common.

Conclusions: Age at HT presentation may influence autoimmune diseases clustering, favoring the association of specific NTADs in different ages of life. Moreover, the association between HT and NTADs increases with age and occurs most frequently in adults.

\section{Introduction}

Hashimoto's thyroiditis (HT) is worldwide the most prevalent autoimmune thyroid disease at any age, and its incidence has been increased in the last decades $(1,2,3)$. It represents a prototypical organ-specific autoimmune disorder, in which environmental and existential factors trigger the development of the immune response against thyroid antigens in genetically susceptible individuals $(4,5,6,7)$. The complex interaction between the genetic background
(C) 2017 European Society of Endocrinology Printed in Great Britain and existential/environmental factors also influences the outcome of the disease, that generally proceeds from an underlying autoimmune diathesis, featured by anti-thyroid autoantibodies production, toward subclinical and then overt hypothyroidism, although its presentation can be even with transient thyrotoxicosis $(1,3,8,9)$.

HT is known to cluster with other autoimmune disorders, and the concept of an autoimmune diathesis

Published by Bioscientifica Ltd. 
is widely accepted $(4,5,7,10,11)$. Previous studies have reported an increased prevalence and relative risk of other autoimmune diseases in subjects with HT and their relatives, mainly highlighting the importance of a shared genetic predisposition $(11,12,13)$. A wide spectrum of autoimmune disorders ranging from either endocrine or non-endocrine organ-specific diseases to systemic diseases has been variously described in association with HT $(11,12,13,14,15,16,17,18,19$, 20). Such an association has been firmly established for some disorders (namely pernicious anemia), to the extent that screening recommendations can be made (11), whereas it has been reported in variable percentages for others, including various rheumatological and cutaneous conditions $(12,13,14,15,16,17,18,19$, 20). However, most studies evaluating such associations have examined the prevalence of HT in non-thyroidal autoimmune diseases rather than the reverse $(10,14$, $15,16,17,18,19,20)$. Also, this estimate indicates that beside the shared genetic predisposition, environmental and existential factors could be critical in determining the diversity of autoimmune manifestations in predisposed individuals (11).

The present study was aimed to investigate the clustering of non-thyroidal autoimmune diseases (NTADs) in two cohorts of patients, children/adolescents and adults, who were all affected by HT. The aim of our study was to ascertain, for the first time, if the age at HT presentation might per se influence the aggregation of NTADs in patients with the same autoimmune disorder (namely HT) by conditioning a different clustering in pediatric and adult age.

\section{Subjects and methods}

\section{Patients}

In this cross-sectional study, we enrolled 1053 newly diagnosed HT patients (500 adults aged 19-80 years and 553 children/adolescents aged 3-18 years) who consecutively referred to the outpatient clinic of the Endocrinology and Paediatrics' Division respectively, of our University Hospital from September 2012 to September 2015. Subjects affected by any syndromic disease (i.e. Trisomy 21, Turner syndrome, Klinefelter syndrome, autoimmune polyglandular syndromes type 1 and type 2) were excluded, as well as were related subjects, to avoid selection bias.

HT was diagnosed according to the currently accepted laboratory and ultrasonographic criteria (serum anti-thyroid antibodies positivity and heterogeneous echo-structure with diffuse or patchy hypoechogenicity at US) (1). Concerning the functional status, individuals with a TSH $>5 \mu \mathrm{IU} / \mathrm{mL}$ at the time of diagnosis were defined hypothyroid and further subdivided into subclinical and overt hypothyroid according to the TSH value being below or above $10 \mu \mathrm{IU} /$ $\mathrm{mL}$ respectively, whereas thyrotoxicosis was defined as undetectable $(<0.01 \mu \mathrm{U} / \mathrm{mL})$ or low $(<0.4 \mu \mathrm{U} / \mathrm{mL}) \mathrm{TSH}$ with elevated free thyroid hormones (21).

All HT patients included in the study were evaluated for non-thyroidal autoimmune diseases (NTADs) by means of careful recording of medical history, physical examination and assessment of selected autoantibody profiles. A number of patients have already been diagnosed for the NTAD prior to HT, but both diseases were diagnosed in the same period of life (adulthood or childhood/adolescence). Patients in whom HT was discovered in adulthood, whereas T1DM or CD, for instance, had been diagnosed in childhood were excluded. All the other subjects were screened for the following non-organ-specific autoantibodies, commonly utilized in rheumatologic practice: antinuclear (ANA), double-stranded (native) DNA (nDNA) and extractable nuclear antigens (ENA) antibodies. Additional specific autoantibodies (i.e. anti-centromere, anti-mitochondrial (AMA), liverkidney microsomal (LKM), antibodies) were searched when appropriate. Rheumatic disorders, such as arthropathies and connective tissue diseases, were diagnosed on the basis of both clinical and laboratory evidence, according to the currently accepted criteria $(22,23,24)$. Also, we assayed sera of each cohort for the following organ-specific autoantibodies: antigastric mucosa (anti-PCA), anti-adrenal cortex (ACA) and anti-21-hydroxylase (21-OHAb), anti-endomysium (EMA) and anti-tissue transglutaminase IgA (aTTG). Diagnosis of coeliac disease (CD) in patients who tested positive for the specific autoantibodies was confirmed by the small intestine biopsy (25). Liver biopsy was critical for the diagnosis of autoimmune hepatitis, as it was mucosal biopsy during colonoscopy for ulcerative colitis (26). Type 1 diabetes (T1DM) screening included determination of anti-glutamic acid decarboxylase (GADA), anti-insulin (IAA) and anti-islet cell (ICA) autoantibodies, as well as glycosylated hemoglobin. Oral glucose tolerance test was performed in patients with autoantibody-positive values (27). Diagnosis of dermatological diseases was based only on clinical features $(28,29,30)$. 
The study design was approved by the Ethics Committee of our hospital, and all the adult patients and the children's parents gave informed written consent to participate.

\section{Methods}

Serum TSH and free thyroxine (FT4) concentrations, as well as anti-thyroglobulin (TgAb) and anti-thyroid peroxidase antibodies (TPOAb), were measured by electrochemiluminescence immunoassay (ECLIA), using commercial kits for Elecsys 1010/2010 e modular analytics E170 supplied by Roche Diagnostics. AntiTSH receptor antibodies (TRAbs) were measured by radioimmunoassay (Brahms Trak Human RIA, supplied by Dasit SpA). Normal values in our laboratory were TSH, 0.27-4.2 $\mu \mathrm{IU} / \mathrm{L} ; \quad$ FT4, 12.0-22.0 pmol/L; TgAb, 0-115 IU/mL; TPOAb, 0-34 IU/mL; TRAb, <1 IU/L. However, it should be considered that the pediatric reference intervals for TSH are wider than those in the adult population with an upper limit up to $6.26 \mathrm{U} / \mathrm{L}$ (3). The aforementioned non-organ- and organspecific autoantibodies were routinely measured by radioimmunoassay or by immunofluorescence or fluoro-enzyme immunoassay with commercial kits, according to the manufacturer's instructions. All samples were processed centrally in the laboratory of our University Hospital of Messina. For all assays, the intra- and the inter-assay CVs were $<5 \%$ and $<10 \%$ respectively.
Thyroid ultrasonography (US) was performed in all patients at the first evaluation using a real-time 2D apparatus (General Electric Healthcare, UK), with a 7.5$10 \mathrm{MHz}$ linear transducer at the Radiology Unit of our University Hospital of Messina.

\section{Statistical analysis}

Numerical data are expressed as mean and standard deviation (s.D.), and categorical variables as number and percentage.

To assess the association between cohorts (adults or children/adolescents) and other categorical variables, such as sex, type of comorbidity and functional status (euthyroidism, subclinical and overt hypothyroidism and thyrotoxicosis), we estimated chi-square test and the relative significance. The same test was applied to compare patients with and without associated NTADs for all the examined categorical variables, for adults and children/ adolescents, separately and, also, to compare the global prevalence of comorbidities in both cohorts.

For NTADs affecting at least 6 patients, we could calculate median age at diagnosis and relative interquartile range. We compared age among the 12 selected NTADs using Kruskal-Wallis test; as it resulted to be highly significant, we performed pairwise comparisons between the NTADs for age using MannWhitney test. For these multiple comparisons, we had to apply Bonferroni's correction, for which the significance alpha level .050 has to be divided by the number of

Table 1 General characteristics of the study population. Data are expressed as mean \pm s.D., median in italics. Normal values are specified under 'Materials and methods' section.

\begin{tabular}{lc}
\hline Parameters & Adults $(n=500)$ \\
\hline Age (year) & \\
Mean \pm S.D. (range) & $40.22 \pm 13.72(19-80)$ \\
Sex & \\
Female & $367(93.4 \%)$ \\
Male & $14.5: 1$ \\
F:M ratio & \\
Functional status $(n(\%))$ & $285(57 \%)$ \\
Euthyroidism & $215(43 \%)$ \\
Hypothyroidism & $146(29.2 \%)$ \\
Subclinical & $69(13.8 \%)$ \\
Overt & 0 \\
Hyperthyroidism & $5.75 \pm 13.03,2.48$ \\
TSH ( $\mu$ IU/L) & $13.87 \pm 3.55,14.0$ \\
FT4 (pmol/L) & $899.95 \pm 3448.25,165.0$ \\
Tg-Ab (IU/L) & $7444.85 \pm 92176.49,216.50$ \\
TPO-Ab (IU/L) &
\end{tabular}

\begin{tabular}{ccc}
\hline Children/adolescents $(n=553)$ & & $\boldsymbol{P}$ value \\
\cline { 1 - 1 } $11.11 \pm 2.96(3-18)$ & - \\
$449(81.2 \%)$ & $<\mathbf{0 . 0 0 0 1}$ \\
$104(18.8 \%)$ & \\
$4.3: 1$ & \\
$300(54.3 \%)$ & \\
$217(39.2 \%)$ & 0.413 \\
$95(17.20 \%)$ & $<.234$ \\
$122(22 \%)$ & \\
$36(6.5 \%)$ & - \\
$13.02 \pm 29.07,4.0$ & $<\mathbf{0 . 0 0 0 1}$ \\
$13.85 \pm 6.53,13.3$ & 0.952 \\
$826.86 \pm 2041.40,237.0$ & 0.673 \\
$1237.71 \pm 2622.60,554.0$ & 0.114 \\
\hline
\end{tabular}

$P$-Values typed in bold are significant $(P \leq 0.05)$. 
the possible seventy-eight pairwise comparisons, so the new 'adjusted' significance level for this analysis is equal to $.050 / 78=.0006$.

Moreover, to evaluate the risk of each NTAD for adult population, odds ratio and relative $95 \%$ confidence interval was calculated.

Statistical analyses were performed using SPSS 17.0 for Window package. $P<0.05$ two sides was considered to be statistically significant.

\section{Results}

\section{General characteristics of adult and pediatric study population}

We compared the data from 500 adult patients (467 F, $33 \mathrm{M}$, mean age at presentation $40.2 \pm 13.7$ years) with those from 553 children/adolescents (449 F, 104 M, mean age at presentation $11.1 \pm 2.9$ years). The analytic results of both groups are presented in Table 1.
The female sex was more represented in both groups, but such a female prevalence was significantly more evident in the adults $(P<0.0001)$.

Concerning thyroid functional status, more than half of the patients were euthyroid at the first evaluation, and about forty percent were hypothyroid, without differences between the two cohorts (Table 1). However, mean serum TSH was significantly higher in children/adolescents than that in adults because the rate of overt hypothyroidism largely exceeded the rate of subclinical hypothyroidism in the former $(P<0.0001)$. Also, the wider serum TSH reference intervals for pediatric age should be considered.

In the pediatric cohort, there were 36 patients $(6.5 \%)$ with transient thyrotoxicosis (Hashitoxicosis) at presentation. In such patients, serum TRAbs remained undetectable during the overall follow-up period, whereas TPOAb serum levels were positive, and hyperthyroidism resolution occurred spontaneously within 3-18 months, with no relapses. No hyperthyroid individuals were found in the adult cohort (Table 1).

Table 2 Prevalence of associated non-thyroidal autoimmune diseases (NTADs) in adult and pediatric/adolescent HT patients.

\begin{tabular}{|c|c|c|c|}
\hline Non-thyroidal autoimmune diseases & Adults & Children/adolescent & $P$ value* \\
\hline & $147 / 500(29.4 \%)^{\S}$ & $104 / 553(18.8 \%)^{\S}$ & $<0.0001$ \\
\hline Arthropathies & $65(13 \%)$ & $1(0.18 \%)$ & $<0.0001$ \\
\hline SpondyloArthritis (SpA) & $40(8 \%)$ & 0 & $<0.0001$ \\
\hline Psoriatic Arthrtitis & $29(5.8 \%)$ & 0 & \\
\hline Other SpA & $11(2.2 \%)$ & 0 & \\
\hline Reumatoid arthritis (RA) & $25(5 \%)$ & $1(0.18 \%)$ & $<0.0001$ \\
\hline Connective tissue diseases & $48(9.6 \%)$ & $1(0.18 \%)$ & $<0.0001$ \\
\hline Sjogren's syndrome (SS) & $28(5.6 \%)$ & 0 & $<0.0001$ \\
\hline Systemic Lupus Erythematosus (SLE) & $7(1.4 \%)$ & 0 & 0.062 \\
\hline Polymyositis & $1(0.2 \%)$ & 0 & \\
\hline Systemic scleroderma (SSc) & $1(0.2 \%)$ & 0 & \\
\hline Undifferentiated connective disease & $10(2 \%)$ & 0 & 0.017 \\
\hline Vasculitis & $1(0.2 \%)$ & $1(0.18 \%)$ & 0.636 \\
\hline Cutaneous diseases & $29(5.8 \%)$ & $25(4.5 \%)$ & 0.507 \\
\hline Vitiligo & $14(2.8 \%)$ & $15(2.7 \%)$ & 0.319 \\
\hline Chronic urticaria & $5(1 \%)$ & $1(0.18 \%)$ & 0.408 \\
\hline Psoriasis & $4(0.8 \%)$ & $2(0.36 \%)$ & 0.991 \\
\hline Erythema nodosum & $3(0.6 \%)$ & 0 & \\
\hline Alopecia & $1(0.2 \%)$ & $5(0.9 \%)$ & 0.091 \\
\hline Lichen & $2(0.4 \%)$ & 0 & \\
\hline Atopic dermatitis & 0 & $2(0.36 \%)$ & \\
\hline Type 1 diabetes mellitus & $2(0.4 \%)$ & $38(6.9 \%)$ & $<0.0001$ \\
\hline Addison's disease & $5(1 \%)$ & $4(0.7 \%)$ & 0.487 \\
\hline Multiple sclerosis & $3(0.6 \%)$ & 0 & 0.381 \\
\hline Gastro-enteric diseases & $15(3 \%)$ & $40(7.23 \%)$ & $<0.0001$ \\
\hline Celiac disease (CD) & $9(1.8 \%)$ & $40(7.23 \%)$ & $<0.0001$ \\
\hline Autoimmune hepatitis & $4(0.8 \%)$ & 0 & \\
\hline Ulcerative colitis (UC) & $2(0.4 \%)$ & 0 & \\
\hline
\end{tabular}

* $P$ values typed in bold are significant $(P \leq 0.05)$. ${ }^{\S}$ Forty adults and 7 pediatric patients had two or more associated NTAD $(P<0.0001)$. NTAD preceded the diagnosis of HT in 70 adults (16 SpA, 14 RA, 7 SS, 4 SLE, 1 polymyositis, 5 undifferentiated connective disease, 10 vitiligo, 1 chronic urticaria, 1 psoriasis, 2 erytema nodosum, 1 multiple sclerosis, 5 CD, 1 UC) and in 75 children/adolescents (36 T1DM, 29 CD, 8 vitiligo, 2 alopecia); $P<0.0001$ adults vs children/adolescents. 
In summary, at HT presentation, both euthyroidism and hypothyroidism were equally frequent in the two cohorts, but the proportions of subclinical and overt hypothyroidism were significantly different, and thyrotoxicosis was definitely more frequent in the pediatric group.

\section{Association with NTADs in adults and children}

The whole spectrum of autoimmune disorders that were found in association with HT in our study population, and their frequencies, are reported in Table 2. As shown, the prevalence of patients with associated NTADs was significantly higher in adults than that in the pediatric cohort: 29.4 vs $18.8 \%(P<0.0001)$. Moreover, the number of adult patients who suffered from two or more associated NTAD was significantly higher than that of children/ adolescents (40 vs $7 ; P<0.0001$ ).

The epidemiological distribution of NTADs was strongly different in the two cohorts. In fact, in the adult patients the most frequent associated diseases were arthropathies, mostly psoriatic arthritis and rheumatoid arthritis (RA), and connective tissue diseases, mostly Sjögren syndrome (SS). In the pediatric/adolescent population, these disorders were absent or rarely found, and the most prevalent autoimmune comorbidities were represented by CD and T1DM (Table 2). Skin diseases were diagnosed in both cohorts with a similar prevalence, vitiligo being the most common. Also, Addison's disease occurred in the two cohorts with a similar prevalence (Table 2).

Such a different clustering of autoimmune disorders in relation with age was even more evident when we stratified all HT patients by age (median and interquartile range) in relation to the type of associated comorbidity (Table 3). When comparing median age at diagnosis between cases with different autoimmune diseases, those with coexisting arthropathies were significantly older compared with patients with no coexisting autoimmune diseases $(P<0.00001)$, as well as compared to those suffering from T1DM, vitiligo, alopecia or CD $(P<0.0001)$. Similarly, subjects affected by connective diseases were older compared to cases without any associated autoimmune disease $(P<0.001)$ and to those with coexisting T1DM or CD $(P<0.001)$ (Table 3$)$. Also, HT patients with comorbidities, as a whole, were older than those without any other autoimmune disease $(P<0.0001)$.

In addition to the differences in aggregation based on age, there was also a different pattern in regard to the presenting autoimmune disease. HT preceded the diagnosis of NTAD in $77(52 \%)$ adults and $29(28 \%)$ children/adolescents $\left(\chi^{2}=14.98 ; P<0.0001\right)$. Within the pediatric/adolescent group, T1DM and CD were usually diagnosed before HT $(P<0.0001)$, whereas HT usually preceded the diagnosis of connective tissue diseases in adults $(P=0.0041)$. In our adult cohort, arthropathies were

Table 3 Median age at HT presentation and interquartile range (years) in all patients (adults plus children/adolescents), in relationship with the most prevalent comorbidities.*

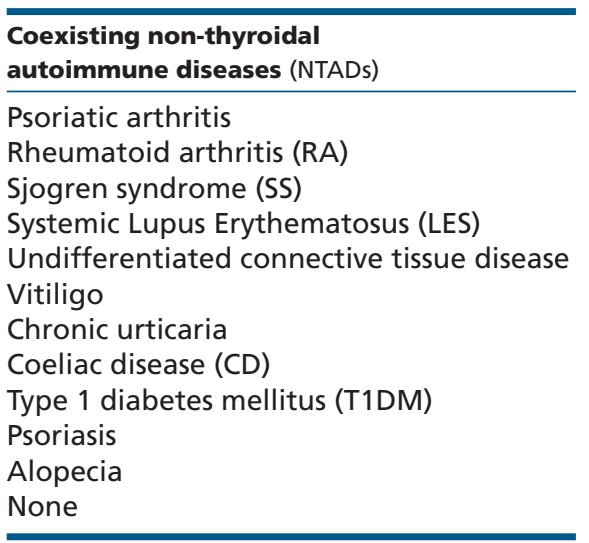

\begin{tabular}{c}
\hline Patients $\mathbf{n}$ \\
\hline 29 \\
26 \\
28 \\
7 \\
10 \\
29 \\
6 \\
49 \\
40 \\
6 \\
6 \\
802
\end{tabular}

\begin{tabular}{c}
\hline Median age at presentation \\
\hline 46 \\
50.5 \\
49 \\
32 \\
41.5 \\
12.9 \\
33 \\
14.6 \\
11.9 \\
15.5 \\
12 \\
14.6
\end{tabular}

\begin{tabular}{c}
\hline Interquartile range \\
\hline $37-57$ \\
$40.7-63$ \\
$42.2-60.5$ \\
$20-34$ \\
$35-50$ \\
$9.2-38$ \\
$18.4-46$ \\
$11.4-16.8$ \\
$9-14$ \\
$9.4-21$ \\
$10.2-14.8$ \\
$10.8-36$
\end{tabular}

The following statistically significant comparisons were recorded: Psoriatis arthritis vs vitiligo, CD, T1DM, psoriasis and alopecia $(P<0.0001)$; RA vs vitiligo, CD and T1DM $(P<0.0001)$, and alopecia $(P=0.0004)$; SS vs LES $(P=0.0004)$, vitiligo, CD and T1DM $(P<0.0001)$, psoriasis $(P=0.002)$, alopecia $(P=0.0001)$; LES vs T1DM $(P=0.0001)$; undifferentiated connective tissue disease vs CD and T1DM $(P<0.0001)$. Data for patients with no other autoimmune comorbidities were also displayed. Comparing patients affected by each of the aforementioned rheumatic disorders with patients without NTADs, the difference in median age was significant $(P<0.0001)$. It was highly significant also the comparison between the median age of patients with NTADs as a whole and that of patients without any comorbidity $(P<0.0001)$. *We performed pairwise comparisons between median ages of patients affected by different NTADs using Mann-Whitney test. Only NTADs affecting six or more patients were included, to allow calculation of interquartile range. According to Bonferroni's correction, the 'adjusted' significance level is $0.050 / 78=0.0006$. 
Table 4 Demographic and clinical parameters of adults and children/adolescents with HT and associated non-thyroidal autoimmune diseases (NTADs).

\begin{tabular}{l}
\hline Parameters \\
\hline Patients with NTAD \\
Age (year) \\
Mean \pm S.D. (range) \\
Sex \\
Female \\
Male \\
F:M ratio \\
Functional status $(n(\%))$ \\
Euthyroidism \\
Hypothyroidism \\
$\quad$ Subclinical \\
$\quad$ Overt \\
Thyrotoxicosis \\
TSH ( $\mu$ IU/L)* \\
FT4 (pmol/L)* \\
Tg-Ab (IU/L)* \\
TPO-Ab (IU/L)*
\end{tabular}

\begin{tabular}{c}
\hline Adults \\
\hline $\mathbf{1 4 7 / 5 0 0 ( 2 9 . 4 \% )}$ \\
$43.40 \pm 14.13(18-80)$ \\
$142(96.6 \%)$ \\
$5(3.4 \%)$ \\
$28: 01: 00$ \\
$86(58.5 \%)$ \\
$61(41.5 \%)$ \\
$43(29.25 \%)$ \\
$18(12.25 \%)$ \\
0 \\
$5.54 \pm 11.79,2.38$ \\
$13.10 \pm 4.76,13.72$ \\
$325.41 \pm 973.09,113$ \\
$340.66 \pm 489.69,187.75$
\end{tabular}

\begin{tabular}{ccc}
\hline Children/adolescent & & $\boldsymbol{P}$ value \\
\cline { 1 - 1 } & & $<\mathbf{0 . 0 0 0 1}$ \\
$11.13 \pm 3.4(2.8-18)$ & - \\
$77(74 \%)$ & $<\mathbf{0 . 0 0 0 1}$ \\
$27(26 \%)$ & \\
$2.8: 1$ & \\
& \\
$69(66.4 \%)$ & 0.256 \\
$28(26.9 \%)$ & $\mathbf{0 . 0 2 5}$ \\
$15(14.4 \%)$ & 0.188 \\
$13(12.5 \%)$ & \\
$7(6.7 \%)$ & - \\
$8.56 \pm 24.85,2.92$ & \\
$15.24 \pm 4.98,14.75$ & \\
$1103.20 \pm 3830.98,175$ & $\mathbf{0 . 0 0 0 1}$ \\
$1359.80 \pm 4255.29,386$ & $\mathbf{0 . 0 1 9}$ \\
&
\end{tabular}

*Data are expressed as mean \pm s.D., median in italics. Normal values are specified under 'Materials and methods' section. $P$ values typed in bold are significant $(P \leq 0.05)$

diagnosed before or after HT at the same extent $(P=0.403)$, as well as $\mathrm{CD}(P=0.8531)$. Skin diseases were diagnosed before or after HT at the same extent both in children $(P=0.2363)$ and adults $(P=0.9484)$, without differences between age groups $(P=0.3899)$.

Comparing the two subgroups of adult and pediatric/ adolescent patients with associated NTADs (Table 4), we found that the female sex was more represented in the two subgroups of patients with associated NTADs, but once again, such a female prevalence was more evident in the adult cohort, indicating that autoimmunity tends to involve mainly women with increasing age. Concerning thyroid functional status, hypothyroidism was found in a higher percentage of adults compared to children/adolescents with comorbidities ( $41.5 \%$ vs $26.9 \%$; $P=0.025$ ).

Comparing in each cohort the HT patients with and without NTADs, some differences emerged. In adults, subjects with associated NTADs were significantly older than those without comorbidities $(43.40 \pm 14.13$ vs $38.9 \pm 13.35$ year; $P<0.0001$ ), with a F:M ratio $28: 1$ in the former and 12:1 in the latter. Thus, adults with comorbidities were more frequently females and older than those without NTADs. In the pediatric/adolescent cohort, there were no differences in age at presentation between subjects with or without NTADs, but the F:M ratio was significantly lower in the former (2.8:1 vs 4.9:1; $P=0.046$ ); thus, males were more represented among children/adolescents with comorbidities. Moreover, subdividing these patients according to their pubertal status, we did not find any significant difference in the prevalence of NTADs between pubertal (69/363 or 19\%) and pre-pubertal $(35 / 190$ or $18 \%)$ subjects $(P=0.866)$.

As shown in Table 5, the relative risk, expressed as odds ratio (OR) of developing an additional autoimmune disorder was greater in adults than in children/ adolescents, and in adulthood it was even higher than the OR of having more than two NTADs. Among the various NTADs, the relative risk was the greatest for RA (OR 21.107), suggesting a strong disease association (Table 5).

Also, the observed prevalence rates of NTADs were compared to those reported in the Italian population, when data were available either from the current

Table 5 Relative risk (odds ratio) of the single non-thyroidal autoimmune disease, comparing prevalence in adult vs pediatric/adolescent cohort (for prevalence values $>0$ ). *

\begin{tabular}{l} 
Coexisting non-thyroidal \\
autoimmune disease (NTAD) \\
\hline Any NTAD \\
Two or more NTAD \\
Rheumatoid arthritis (RA) \\
Vasculitis \\
Vitiligo \\
Chronic urticaria \\
Type 1 diabetes mellitus (T1DM) \\
Coeliac disease (CD) \\
Psoriasis \\
Alopecia
\end{tabular}

\begin{tabular}{ccc}
$\begin{array}{ccl}\text { Odds } \\
\text { ratio }\end{array}$ & & $\begin{array}{c}95 \% \text { Confidence } \\
\text { interval }\end{array}$ \\
\cline { 1 - 1 } 1.798 & & $1.349-2.396$ \\
5.18 & & $2.217-12.105$ \\
21.107 & & $2.811-158.465$ \\
0.705 & & $0.044-11.409$ \\
0.625 & & $0.287-1.357$ \\
3.627 & & $0.417-31.511$ \\
0.024 & & $0.006-0.102$ \\
0.104 & & $0.048-0.228$ \\
1.427 & & $0.256-7.937$ \\
0.136 & & $0.016-1.179$ \\
\hline
\end{tabular}

*OR was calculated for all autoimmune comorbidities, which were represented in both groups. 
literature $(31,32,33,34)$ or from our Health Department (www.salute.gov.it). Most of the NTADs were reported more frequently in our HT subjects than in the general population: psoriatic arthritis, $5.8 \%$ vs $1 \%$ in the general population; RA, $5 \%$ vs $1 \%$; SS, $5.6 \%$ vs $3 \%$; LES, $1.4 \%$ vs $0.07 \%$; T1DM in children/adolescents, $6.8 \%$ vs $1.5 \%$; CD $1.8 \%$ vs $0.7 \%$ in adults, $7.2 \%$ vs $1.25 \%$ in children/ adolescents; vitiligo, $2.8 \%$ in adults and $2.7 \%$ in children/ adolescents vs $2.0 \%$ in the general population. Although limited by the paucity of contemporary population prevalence data, our data support the presence of a true, non-casual disease association and an increased risk of other autoimmune disease in HT patients, mainly for connective tissue diseases in adult age, for T1DM in pediatric age and for CD and vitiligo in both age groups.

\section{Discussion}

In the present study, we have evaluated the association between HT and other autoimmune diseases in more than 1000 well-characterized, newly diagnosed HT patients at any age of life to delineate the clinical patterns of diseases aggregation in relation with age. To the best of our knowledge, this is the largest cohort so far reported in the literature, which includes even pediatric/adolescent patients along with adults with HT.

It is currently known that increasing age and female gender are associated with increasing risk of developing autoimmune disorders $(7,10)$, but scanty data are available on their possible role in conditioning the aggregation of autoimmune diseases. The present study was aimed to ascertain whether the age at HT presentation might per se influence the aggregation of NTADs in patients with the same autoimmune disorder (namely HT) by conditioning a different clustering of diseases in pediatric/adolescent and adult age.

A previous multicenter study by Boelaert and coworkers (13) have estimated the prevalence and the relative risk of other autoimmune diseases in a wellcharacterized cohort of adult Caucasian subjects (mean age 43 years) suffering from HT $(n=495)$ or Graves' disease $(n=2791)$. The estimated prevalence of another NTAD was $14.3 \%$ in HT and $9.67 \%$ in Graves' disease cases, and the relative risks of all other coexisting autoimmune disorders were significantly increased in both. In this cohort, even if women definitely outnumbered men (2744 F and $542 \mathrm{M}$ ), there were no significant differences in the prevalence of associated NTADs between men and women. In the same manner, no differences in age at diagnosis were reported between HT cases with or without associated NTADs, whereas Graves's disease cases with coexisting RA and pernicious anemia were older than cases without any associated NTADs. However, comparison between the whole group of patients with different NTADs and those without any associated NTAD was not provided in the study (13). Thus, the study from Boelaert and coworkers provided strong evidence for true diseases association, but no additional information about the possible influence of age on such an association as it was beyond the aim of the study.

In the present study on a large cohort, we not only just collected the clinical records but also screened all the participants for common autoimmune diseases. Furthermore, unlike previous studies, our HT population consisted of both adults ( 550 cases, comparable by number, sex and age to the study by Boelaert and coworkers) and pediatric/adolescent (553 cases, aged <18 years) patients. This has an important significance for the diseases prevalence estimates as some of the NTADs investigated tend to occur early in life (i.e. T1DM and CD), whereas others tend to occur later (i.e. RA).

In our study, the prevalence of associated NTADs in the whole cohort of HT subjects was 23.8\%, and it was significantly higher in adults. Also the number of patients suffering from two or more associated NTADs was significantly higher in adults than in children/ adolescents. Moreover, subjects with associated NTADs were significantly older than those without comorbidities. The relative risk of developing NTADs increased with age as adults had a nearly twofold risk compared to children/ adolescents. Thus, we report an increasing prevalence of coexisting NTADs with age, as well as an increased OR for one or more other autoimmune disorders.

Female subjects were more frequently affected by autoimmune comorbidities with increasing age. However, such a female preponderance was not evident in children/ adolescents. Under the age of eighteen, the female: male ratio was significantly lower in HT patients with NTADs compared to those without comorbidities, suggesting that sex-related factors are less important in conditioning autoimmune diseases aggregation at younger age. Accordingly, we did not find any difference in NTADs prevalence when subdividing these subjects according to their pubertal status.

Although most disease associations described in the present study have already been reported individually in the literature, the present data provide novel information about their epidemiological distribution and clustering, and first highlight that there are significant differences 
in the prevalence and peculiar aggregations of NTADs in different ages. In fact, the NTADs distribution was strongly different in the two cohorts. In adults, the most frequently associated diseases were arthropathies (psoriatic arthritis and RA) and connective tissue diseases (mostly SS), and the OR was the greatest for RA (more than 20-folds). On the contrary, in the pediatric/adolescent cohort, these disorders were absent or rarely found, and the most prevalent ones were T1DM and CD. Skin diseases were represented with similar prevalence in both cohorts, vitiligo being the most common. Accordingly, when comparing median age at diagnosis between cases with different NTADs, patients with coexisting arthropathies and/or connective diseases were significantly older compared with those reporting T1DM, vitiligo, alopecia or CD.

Very recently, similar findings have been reported in another organ-specific autoimmune disease that often clusters with HT, i.e. CD (20). In a retrospective study including more than $400 \mathrm{CD}$ patients, the occurrence of another autoimmune disease was reported in $25.2 \%$ of the patients (the most prevalent being T1DM, microscopic colitis and autoimmune thyroid diseases), and the age of CD diagnosis was significantly higher in patients with a coexisting autoimmune disorder compared to those with CD alone $(P=.002)$. Older age at the time of CD diagnosis, and not the time of follow-up or the disease duration, was an independent predictor of having a concomitant autoimmune disorder. The authors hypothesized that CD diagnosis later in life, thus possibly prolonged inflammation, may favor the occurrence of other autoimmune disorders (20).

It is conceivable that, beside the genetic background, autoimmune disorders may share common exogenous factors potentially involved in the development and progression of the disease, such as vitamin D deficiency, increased oxidative stress, improved hygiene and intestinal microbiota dysbiosis, whose role in autoimmunity is intensely debated at present $(6,7,35,36,37)$. Specially, there is growing evidence on the role of microbiome in various age-related disorders, including rheumatoid arthritis and spondyloarthritis (37). This hypothesis may account for the high rate of association between HT and rheumatic diseases with increasing age in our cohort.

In conclusion, the present study confirms a high prevalence of NTADs in HT patients, and for the first time, delineates different patterns of aggregation of autoimmune disorders in relation to age in these patients. Age at presentation of HT may influence autoimmune diseases clustering, favoring the association of some specific NTADs, such as T1DM and CD in children/ adolescents and rheumatic diseases in adulthood. As a consequence, different types of NTADs should be investigated/suspected in relation with patient's age. Moreover, the association between HT and NTADs increases with age, and adult HT subjects have both increased prevalence and risk of concomitant NTADs in comparison with children/adolescents. Common agerelated autoimmune mechanisms may contribute to the pathogenesis of coexisting autoimmune diseases.

\section{Declaration of interest}

The authors declare that there is no conflict of interest that could be perceived as prejudicing the impartiality of the research reported.

\section{Funding}

This research did not receive any specific grant from any funding agency in the public, commercial or not-for-profit sector.

\section{References}

1 Caturegli P, De Remigis A \& Rose NR. Hashimoto thyroiditis: clinical and diagnostic criteria. Autoimmunity Reviews 201413 391-397. (doi:10.1016/j.autrev.2014.01.007)

2 McLeod DS \& Cooper DS. The incidence and prevalence of thyroid autoimmunity. Endocrine 201542 252-265. (doi:10.1007/s12020-0129703-2)

3 Wasniewska M, Corrias A, Salerno M, Mussa A, Capalbo D, Messina MF, Aversa T, Bombaci S, De Luca F \& Valenzise M. Thyroid function patterns at Hashimoto's thyroiditis presentation in childhood and adolescence are mainly conditioned by patients' age. Hormone Research in Paediatrics 201278 232-236. (doi:10.1159/000343815)

4 Weetman AP. The genetics of autoimmune thyroid disease. Hormone Metabolic Research 200941 421-425. (doi:10.1055/s-0029-1214415)

5 Effraimidis G \& Wiersinga WM. Mechanisms in endocrinology: autoimmune thyroid disease: old and new players. European Journal of Endocrinology 2014170 R241-R252. (doi:10.1530/EJE-14-0047)

6 Giovinazzo S, Vicchio TM, Certo R, Alibrandi A, Palmieri O, Campennì A, Cannavò S, Trimarchi F \& Ruggeri RM. Vitamin D receptor gene polymorphisms/haplotypes and serum 25(OH)D3 levels in Hashimoto's thyroiditis. Endocrine 2016. (Epub ahead of print) (doi:10.1007/s12020-016-0942-5)

7 Ajjan RA \& Weetman AP. The pathogenesis of Hashimoto's thyroiditis: further developments in our understanding. Hormone Metabolic Research 201547 702-710. (doi:10.1055/s-0035-1548832)

8 Aversa T, Valenzise M, Corrias A, Salerno M, Mussa A, Capalbo D, Salzano G, De Luca F \& Wasniewska M. Subclinical hyperthyroidism when presenting as initial manifestation of juvenile Hashimoto's thyroiditis: first report on its natural history. Journal of Endocrinological Investigation 201437 303-308. (doi:10.1007/s40618014-0054-0)

9 Wasniewska M, Corrias A, Salerno M, Lombardo F, Aversa T, Mussa A, Capalbo D, De Luca F \& Valenzise M. Outcomes of children with hashitoxicosis. Hormone Research in Paediatrics 201277 36-40. (doi:10.1159/000334640)

10 Weetman AP. Non-thyroid autoantibodies in autoimmune thyroid disease. Best Practice and Research Clinical Endocrinology and Metabolism 200519 17-32. (doi:10.1016/j.beem.2004.11.004) 
11 Weetman AP. Diseases associated with thyroid autoimmunity: explanations for the expanding spectrum. Clinical Endocrinology 2011 74 411-418. (doi:10.1111/j.1365-2265.2010.03855.x)

12 Cooper GS, Bynum ML \& Somers EC. Recent insights in the epidemiology of autoimmune diseases: improved prevalence estimates and understanding of clustering of diseases. Journal of Autoimmunity 200933 197-207. (doi:10.1016/j.jaut.2009.09.008)

13 Boelaert K, Newby PR, Simmonds MJ, Holder RL, Carr-Smith JD, Heward JM, Manji N, Allahabadia A, Armitage M, Chatterjee KV et al. Prevalence and relative risk of other autoimmune diseases in subjects with autoimmune thyroid disease. American Journal of Medicine 2010 123 183.e1-183.e9. (doi:10.1016/j.amjmed.2009.06.030)

14 Punzi L \& Betterle C. Chronic autoimmune thyroiditis and rheumatic manifestations. Joint Bone Spine 200471 275-283. (doi:10.1016/j. jbspin.2003.06.005)

15 Biró E, Szekanecz Z, Czirják L, Dankó K, Kiss E, Szabó NA, Szucs G, Zeher M, Bodolay E, Szegedi G et al. Association of systemic and thyroid autoimmune diseases. Clinical Rheumatology 200625 240-245. (doi:10.1007/s10067-005-1165-y)

16 Acay A, Ulu MS, Ahsen A, Eroglu S, Ozuguz U, Yuksel S \& Acarturk G. Assessment of thyroid disorders and autoimmunity in patients with rheumatic diseases. Endocrine, Metabolic and Immune Disorders: Drug Targets 201414 182-186. (doi:10.2174/18715303146 66140626113111)

17 Doyle EA. Autoimmune conditions associated with type 1 diabetes. Journal of Pediatric Nursing 201541 89-91.

18 Kakourou T, Kanaka-Gantenbein C, Papadopoulou A, Kaloumenou E $\&$ Chrousos GP. Increased prevalence of chronic autoimmune (Hashimoto's) thyroiditis in children and adolescents with vitiligo. Journal of the American Academy of Dermatology 200553 220-223. (doi:10.1016/j.jaad.2005.03.032)

19 Alpigiani MG, Cerboni M, Bertini I, d'Annunzio G, Haupt R, Iester A $\&$ Lorini R. Endocrine autoimmunity in young patients with juvenile chronic arthritis. Clinical and Experimental Rheumatology 200220 $565-568$.

20 Spijkerman M, Tan IL, Kolkman JJ, Withoff S, Wijmenga C, Visschedijk MC \& Weersma RK. A large variety of clinical features and concomitant disorders in celiac disease - a cohort study in the Netherlands. Digestive and Liver Disease 201648 499-505. (doi:10.1016/j.dld.2016.01.006)

21 Biondi B \& Cooper DS. The clinical significance of subclinical thyroid dysfunction. Endocrine Reviews 200829 76-131. (doi:10.1210/er.20060043)

22 Firestein GS, Budd RC, Gabriel SE, McInnes IB \& O'Dell JR. Kelley's Textbook of Rheumatology, 9th Edition. Philadelphia, PA, USA: Saunders, 2013.

23 Aletaha D, Neogi T, Silman AJ, Funovits J, Felson DT, Bingham CO 3rd, Birnbaum NS, Burmester GR, Bykerk VP, Cohen MD et al. Rheumatoid arthritis classification criteria: an American College of Rheumatology/European League Against Rheumatism collaborative initiative. Arthritis Rheumatoid 201062 2569-2581. (doi:10.1002/art.27584)

24 Brito-Zerón P, Theander E, Baldini C, Seror R, Retamozo S, Quartuccio L, Bootsma H, Bowman SJ, Dörner T, Gottenberg JE et al.
Early diagnosis of primary Sjögren's syndrome: EULAR-SS task force clinical recommendations. Expert Review of Clinical Immunology 2016 12 137-156. (doi:10.1586/1744666x.2016.1109449)

25 Editors Internal Clinical Guidelines Team (UK) Coeliac Disease: Recognition, Assessment and Management. Source London: National Institute for Health and Care Excellence (UK). National Institute for Health and Care Excellence: Clinical Guidelines, 2015.

26 Qiu D, Wang Q, Wang H, Xie Q, Zang G, Jiang H, Tu C, Guo J, Zhang S, Wang J et al. Validation of the simplified criteria for diagnosis of autoimmune hepatitis in Chinese patients. Journal of Hepatology 201154 340-347. (doi:10.1016/j.jhep.2010.06.032)

27 Pietropaolo M, Peakman M, Pietropaolo SL, Zanone MM, Foley TP Jr, Becker DJ \& Trucco M. Combined analysis of GAD65 and ICA512(IA-2) autoantibodies in organ and non-organ-specific autoimmune diseases confers high specificity for insulin-dependent diabetes mellitus. Journal of Autoimmunity 2008 11 1-10. (doi:10.1006/ jaut.1997.0170)

28 Ezzedine K, Eleftheriadou V, Whitton M \& van Geel N. Vitiligo. Lancet 2015386 74-84. (doi:10.1016/S0140-6736(14)60763-7)

29 Islam N, Leung PS, Huntley AC \& Gershwin ME. The autoimmune basis of alopecia areata: a comprehensive review. Autoimmune Reviews 201514 81-89. (doi:10.1016/j.autrev.2014.10.014)

30 Ruggeri RM, Imbesi S, Saitta S, Campennì A, Cannavò S, Trimarchi F $\&$ Gangemi S. Chronic idiopathic urticaria and Graves' disease. Journal of Endocrinological Investigation 201336 531-536. (doi:10.3275/8940)

31 Ciocci A, Buratti L, Di Franco M \& Mauceri MT. L'epidemiologia delle malattie reumatiche: confronto fra i dati italiani e quelli stranieri. Reumatismo 199951 (Supplement 2) 201.

32 Benucci M, Del Rosso A, Li Gobbi F, Manfredi M, Cerinic MM \& Salvarani C. Systemic lupus erythematosus (SLE) in Italy: an Italian prevalence study based on a two-step strategy in an area of Florence (ScandicciLe Signe). Medical Science Monitor 200511 CR420-CR425.

33 Mustalahti K, Catassi C, Reunanen A, Fabiani E, Heier M, McMillan S, Murray L, Metzger MH, Gasparin M \& Coeliac EU Cluster, Project Epidemiology. The prevalence of celiac disease in Europe: results of a centralized, international mass screening project. Annals of Medicine 201042 587-595. (doi:10.3109/07853890.2010.505931)

34 Bruno G, Maule M, Merletti F, Novelli G, Falorni A, Iannilli A, Iughetti L, Altobelli E, d'Annunzio G, Piffer S et al. Age-period-cohort analysis of 1990-2003 incidence time trends of childhood diabetes in Italy: the RIDI study. Diabetes 201059 2281-2287. (doi:10.2337/db10-0151)

35 Ruggeri RM, Vicchio TM, Cristani M, Certo R, Caccamo D, Alibrandi A, Giovinazzo S, Saija A, Campennì A, Trimarchi F et al. Oxidative stress and advanced glycation end products in Hashimoto's thyroiditis. Thyroid 201626 504-511. (doi:10.1089/thy.2015.0592)

36 Rosser EC \& Mauri C. A clinical update on the significance of the gut microbiota in systemic autoimmunity. Journal of Autoimmunity 2016 29 S0896-8411(16)30087-7. (doi:10.1016/j.jaut.2016.06.009)

37 Costello ME, Robinson PC, Benham H \& Brown MA. The intestinal microbiome in human disease and how it relates to arthritis and spondyloarthritis. Best Practice and Research Clinical Rheumatology 2015 29 202-212. (doi:10.1016/j.berh.2015.08.001)

Received 29 August 2016

Revised version received 18 October 2016

Accepted 8 November 2016 